\title{
HFS Injection of X-Mode for EBW Conversion in QUEST
}

\author{
Hatem ELSERAFY, Kazuaki HANADA ${ }^{1)}$, Kengoh KURODA ${ }^{1)}$, Hiroshi IDEI ${ }^{1)}$, Ryota YONEDA ${ }^{2)}$,

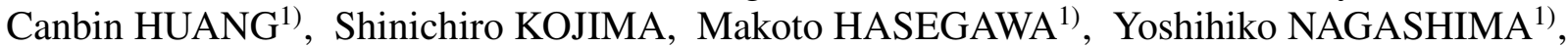 \\ Takumi ONCHI $^{1)}$, Ryuya IKEZOE ${ }^{1)}$, Aki HIGASHIJIMA ${ }^{1)}$, Takahiro NAGATA ${ }^{1)}$, \\ Shoji KAWASAKI ${ }^{1)}$, Shun SHIMABUKURO ${ }^{1)}$, Nicola BERTELLI' ${ }^{3)}$ and Masayuki ONO $^{3)}$ \\ IGSES, Kyushu University, Kasuga, Fukuoka 816-8580, Japan \\ ${ }^{1)}$ TRIAM, Kyushu University, Kasuga, Fukuoka 816-8580, Japan \\ ${ }^{2)}$ National Institute for Fusion Science, Toki 509-5292, Japan \\ 3) Princeton Plasma Physics Laboratory, Princeton, NJ 08540, USA
}

(Received 7 December 2018 / Accepted 7 January 2019)

\begin{abstract}
High field side (HFS) injection of eXtra-ordinary X-mode for electron Bernstein wave (EBW) conversion was conducted in the QUEST tokamak. Radio frequency (RF; 8.2 GHz) power was delivered from the low field side (LFS) to the high field side HFS through waveguides, and from the HFS placed $20 \mathrm{~cm}$ above the midplane of the vacuum vessel. The aim was to compare the RF launches from the LFS and HFS. The plasma brightness, measured by a fast camera, as well as the $\mathrm{H}_{\alpha}$ signal captured along the mid-plane, was noticeably higher in the HFS launch than in the LFS launch. The HFS injection achieved a plasma current of approximately $130 \mathrm{~A}$, versus $35 \mathrm{~A}$ in the LFS injection. The electron density $n_{\mathrm{e}}$ predicted from the position of the upper hybrid resonance agreed with the line-averaged $n_{\mathrm{e}}$ measured by an interferometer, confirming the effective conversion and subsequent damping of the EBW mode. The RF leakage of the HFS injection was less than one-sixth that of the LFS injection. These results indicate that HFS delivers better RF coupling and conversion efficiency to EBW than LFS injection. Such efficient plasma heating via EBW will significantly enhance the plasma production.
\end{abstract}

(c) 2019 The Japan Society of Plasma Science and Nuclear Fusion Research

Keywords: HFS launch, EBW, plasma production

DOI: $10.1585 / \mathrm{pfr} .14 .1205038$

Fusion reactors with low aspect ratio require noninductive methods to minimize the complications of applying a center solenoid in a compact space during plasma startup and current driving. The Q-shu University Experiment with Steady-state Spherical Tokamak (QUEST) is a spherical tokamak with major and minor radii of $0.68 \mathrm{~m}$ and $0.4 \mathrm{~m}$, respectively, and a maximum toroidal field of $0.25 \mathrm{~T}$ at $R=0.64 \mathrm{~m}$ [1]. With a low field side (LFS) injection of radio frequency (RF; $8.2 \mathrm{GHz}$ ) from a fully noninductive current drive (CD) [2,3], QUEST delivered a $1 \mathrm{~h}$ $55 \mathrm{~m}$ discharge. QUEST relies primarily on non-inductive methods such as coaxial helicity injection for the plasma startup and CD, achieving up to $29 \mathrm{kA}$ of toroidal current [4] and $69 \mathrm{kA}$ of plasma current under $28 \mathrm{GHz}$ electron cyclotron heating (ECH) [5]. However, electron Bernstein wave (EBW) [6] has not been previously explored in QUEST. Various EBW conversion modes have been identified. One is the LFS injection of O-mode, which is converted first to X-mode in plasma, then to EBW after reaching upper hybrid resonance (UHR). These conversions are known as the O-X-B scenario [7]. Another scenario is the LFS injection of X-mode that tunnels the cutoff layer, and converts to EBW after reaching the UHR [8]. The current work focuses on the third scenario, the direct conversion of

author'se-mail: elserafy@kyudai.jp
X-mode to EBW by HFS injection. To our knowledge, all previous investigations placed a mirror polarizer at the high field side (HFS) for launching the X-mode, which converts to EBW in the UHR layer. Examples are MAST's 60GHz system [9], WT-3's 56-GHz system [10] and TCA's 39-GHz system [11]. As the RF is lower in QUEST than in the abovementioned systems, a larger reflection mirror should be installed on the HFS's side of the vessel, which is rather difficult. To resolve this problem and properly deliver the RF to the HFS of the QUEST vessel, the waveguides were tentatively installed as shown in Fig. 1.

In this setup, the klystron was connected to the QUEST vacuum vessel by atmospheric waveguides constructed from $\mathrm{Al}$ and $\mathrm{Cu}$. Next, the atmospheric waveguides were connected to the in-vessel waveguides (here referred to as vacuum waveguides) by vacuum flanges. The vacuum waveguides are made of $\mathrm{Cu}$ and are located inside the vessel (see Fig. 1). They are connected along the hot wall [3] and the diverter plate to minimize contact with the plasma. To avoid breakdown in the electron cyclotron resonance layer (ECRL), the vacuum waveguides are filled with $\mathrm{SF}_{6}$ gas. A vacuum window located on the HFS past the ECRL is made of sapphire, which is robust against breakage. In this setup, two waveguides were connected to two klystrons each with a maximum power output of 


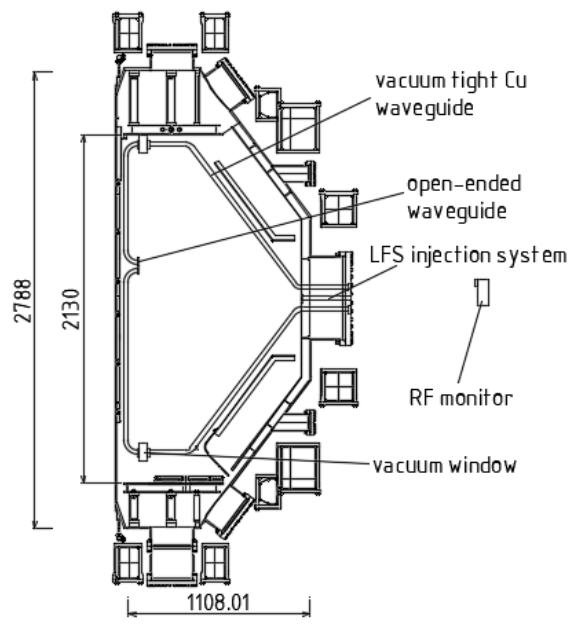

Fig. 1 Experimental setup of RF power transmission from LFS to HFS using waveguides.
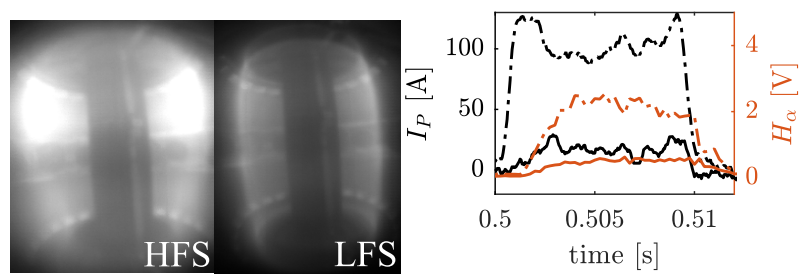

Fig. 2 Left: Fast camera views, Right: $I_{\mathrm{p}}$ (black) and $\mathrm{H}_{\alpha}$ radiation (brown) in LFS (solid) and HFS (dashed) injection at $B_{\mathrm{T}}=0.25 \mathrm{~T}$ and $B_{\mathrm{P}}=0 \mathrm{~T}$.

$25 \mathrm{~kW}$. The antennae positions were displaced $195 \mathrm{~mm}$ above the mid-plane and the waveguides were open-ended.

The experiment was conducted in the absence of a poloidal field $\left(B_{\mathrm{P}}=0 \mathrm{~T}\right)$. Conventionally, the ECRL is set at $R=0.32 \mathrm{~m}$ [12], but here we set the ECRL at $R=0.55 \mathrm{~m}$ (i.e., $B_{\mathrm{T}}=0.25 \mathrm{~T}$ ) to avoid ECRL-antenna contact and consequent arcing. In addition, because no water cooling is installed in the waveguides and the sapphire windows, the baking (usually conducted at $200^{\circ} \mathrm{C}$ ) was maximized at $80^{\circ} \mathrm{C}$ and was regulated at $5^{\circ} \mathrm{C} /$ hour to avoid excess thermal expansion of the $\mathrm{Cu}$ waveguides and to regulate the vacuum connection. The absence of water cooling limits the RF shot pulse to $100 \mathrm{~ms}$. To maintain a wide safety margin, we limited each RF shot pulse to $10 \mathrm{~ms}$.

Figure 2 shows fast camera views captured during the LFS and HFS injections. A direct comparison of the brightness levels confirms that the HFS injection is much brighter than the LFS launch at $12 \mathrm{~kW}$ of injected RF power. The HFS injection also yielded a higher plasma current $\left(I_{\mathrm{p}}=130 \mathrm{~A}\right)$ and a higher Balmer series line radiation $\left(\mathrm{H}_{\alpha}\right)$ than the LFS injection. The edge of the LFS brightness suggests the position of the UHR at which the EBW is producible. Because of the low plasma temperature, the EBW was deposited on the plasma by collisional damping. The large bend of the LFS edge indicates a large shift of the UHR caused by the high density. The UHR position is given by

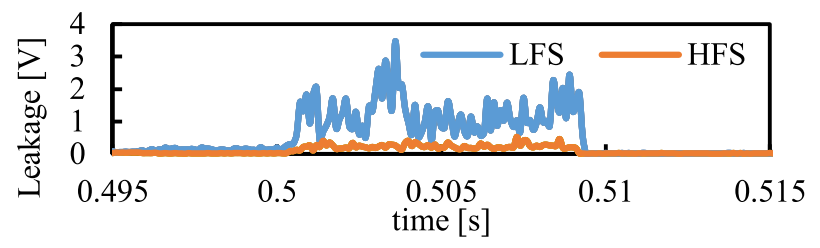

Fig. 3 RF leakage in HFS is $1 / 6$ that of LFS.

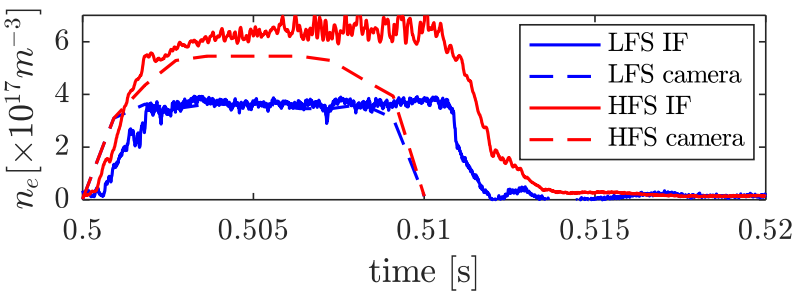

Fig. 4 Density determined from IF and camera images.

$$
R_{\mathrm{UHR}}=R_{\mathrm{ECRL}} \sqrt{\omega_{\mathrm{RF}}^{2} /\left(\omega_{\mathrm{RF}}^{2}-\omega_{\mathrm{pe}}^{2}\right)},
$$

where $\omega_{\mathrm{RF}}, \omega_{\mathrm{pe}}$, and $R_{\mathrm{ECRL}}$ denote the RF frequency, plasma frequency, and major radius of the ECRL, respectively. The superior plasma production in HFS injection suggests an improved conversion efficiency to EBW. This assumption is supported by the RF leakage power, which is measured by monitors surrounding the QUEST vessel. As shown in Fig. 3, the RF leakage was much smaller in the HFS launch than in the LFS injection.

Although the $I_{\mathrm{p}}$ and $\mathrm{H}_{\alpha}$ were comparatively low (see Fig. 2), the HFS and LFS were sufficiently different to conclude that the EBW conversion efficiency is much higher in the HFS injection than in the LFS injection. To confirm the EBW conversion, the plasma density based on Eq. (1) was compared with the line-averaged electron density measured by an interferometer (IF) placed at the midplane. The two results agreed (see Fig. 4), indicating that plasma production around the UHR was successful, and strongly relied on EBW conversion and damping. However, the EBWCD was not obtained, and a much higher RF power and cleaner wall conditions are required to clarify the EBWCD. This improvement will be tackled in future work.

In conclusion, the preliminary results suggest that HFS injection of X-mode through waveguides in QUEST achieves an efficient EBW conversion, opening the way for further EBW investigations such as driving the plasma current and increasing the plasma density.

\section{Acknowledgments}

This work was supported by a Grant-in-Aid for JSPS Fellows (KAKENHI Grant Number 16H02441, 24656559) and the NIFS Collaboration Research Program (NIFS05KUTRO14, NIFS13KUTR093, NIFS13KUTR085, and NIFS14KUTR103). This work was also supported in part by the Collaborative Research 
Program of the Research Institute for Applied Mechanics, Kyushu University.

[1] K. Hanada et al., Plasma Fusion Res. 5, S1007 (2010).

[2] K. Hanada et al., Plasma Sci. Technol. 13, 307 (2011).

[3] K. Hanada et al., Nucl. Fusion 57, 126061 (2017).

[4] K. Kuroda et al., Plasma Fusion Res. 12, 1202020 (2017).
[5] H. Idei et al., Nucl. Fusion 57, 126045 (2017).

[6] I. Bernstein, Phys. Rev. 109, 10 (1958).

[7] R. Yoneda et al., Plasma Fusion Res. 13, 3402115 (2018).

[8] S. Kim et al., Phys. Plasmas 21, 062108 (2014).

[9] V. Shevchenko et al., Nucl. Fusion 50, 022004 (2010).

[10] T. Maekawa et al., Phys. Rev. Lett. 86, 003783 (2001).

[11] D. Whaley et al., Nucl. Fusion 32, 757 (1992).

[12] H. Miura et al., Plasma Fusion Res. 10, 3402066 (2015). 